\title{
EQUIVALENTE DE CIRCUITOS COM MODELAGEM DINÂMICA DE COMPONENTES
}

\author{
Isa Helena C. Carramaschi*, Francisco Damasceno Freitas ${ }^{\dagger}$, Tito Ricardo Vaz da \\ COsTA $\ddagger$ \\ *Venâncio 3000, Bloco A \\ Asa Norte, Brasitia, DF, Brasil \\ ${ }^{\dagger}$ Departamento de Engenharia Elétrica - Faculdade de Tecnologia - Universidade de Brasilia \\ Campus Asa Norte - Asa Norte - Brasilia, DF, Brasil \\ $\ddagger S G A N 603$ Blocos $I$ e $J$ \\ Asa Norte - Brasília, DF, Brasil
}

Emails: isa.helena@eln.gov.br, ffreitas@ene.unb.br, titoricardo@aneel.gov.br

\begin{abstract}
This paper discusses a methodology for computing a dynamical equivalent based on the Thèvenin and Norton equivalents. These equivalents have an active network with a voltage or current source plus an impedance. In general, both of them are computed from steady-state quantities of the network. This may be a constraining in the case of fast transient, such as those ones due to switching in power systems. In the proposed methodology in this paper, we compute a dynamical source which is connected with the equivalent impedance (admittance). in this sense dynamical expressions are deduced and examples are discussed. To demonstrate the performance of the technique, tests are carried out on an electric circuit.
\end{abstract}

Keywords - Dynamical systems, dynamical equivalent, Thèvenin equivalent, electromagnetic transients.

Resumo - Este artigo aborda uma metodologia para o cálculo de equivalente dinâmico com base nos equivalentes de Thèvenin e Norton. O uso destes equivalentes, via de regra, considera uma fonte, com valor de regime estacionário e uma impedância, esta calculada para uma frequência de interesse. Na metodologia proposta, propõe-se uma representação dinâmica, tanto da fonte quanto da impedância do equivalente. Com este objetivo, são desenvolvidas e apresentadas expressões para melhor entendimento da metodologia. Testes em um circuito elétrico ilustram a eficácia da técnica.

Palavras-chave— sistemas dinâmicos, equivalente dinâmico, equivalente de Thèvenin, transitórios eletromagnéticos.

\section{Introdução}

Alguns estudos em várias áreas do conhecimento requerem simulações dinâmicas em que o período de interesse são os instantes que caracterizam a fase transitória. Em sistemas elétricos de potência, estudos desta natureza incluem análise de estabilidade e de transitórios eletromagnéticos. No primeiro tipo, o foco são fenômenos na faixa de 0,1 a 2,5 Hz, os chamados transitórios eletromecânicos. No segundo tipo, o alvo são transitórios de alta frequência, cuja faixa pode se estender desde $\mathrm{Hz}$ até $\mathrm{MHz}$ (Watson and Arrillaga, 2003). Evidentemente, nesta faixa tão ampla de frequência a modelagem do fenômeno é decisiva na simulação.

Na prática, há modelos muito complexos e outros simplificados. Mas, de uma forma geral, diversos modelos podem ser avaliados por meio de modelagem com base em circuitos RLC (Freitas et al., 2011). Este fato é justificado, porque os sistemas são caracterizados por modos de funcionamento. Um modo de operação, por sua vez, pode ser simulado por um circuito RLC desacoplado, controlável e observável.

Este artigo propõe o cálculo de um equivalente dinâmico com base nos resultados oriundos dos equivalentes de Thèvenin e Norton (Johnson D. E., 1994). O método consiste no cálculo de uma fonte que contém tanto valores transitórios, como de regime estacionário, característico do sinal de excitação (Carramaschi, 2010). Na prática, costuma-se calcular apenas as componentes de regime permanente. A metodologia é testada em circuito elétrico RLC, no qual chaveamentos são realizados no sentido a avaliar o desempenho da técnica diante de transitórios. Os testes são realizados no aplicativo ATP (Alternative Transient Program) (Group, 1992) e no Matlab.

Este artigo está organizado da seguinte forma: além da seção introdutória, a Seção 2 aborda os problemas relativos aos equivalentes de Thèvenin e Norton. Na Seção 3, calcula-se a impedância ou admitância do equivalente. Testes e resultados são alvos da Seção 4. Finalmente, dedica-se a Seção 5 para destaque das principais conclusões do trabalho.

\section{Equivalente Dinâmico}

Nesta seção, ilustra-se como é obtido o equivalente de um circuito tendo como base o resultado dos teoremas de Norton e de Thèvenin. O objetivo é calcular as contribuição da fonte do equivalente durante o período transitório e de regime estacionário. O estudo se justifica, porque em algumas simulações de transitórios, como os eletromagné- 
ticos em sistemas elétricos de potência (Watson and Arrillaga, 2003), é usual se considerar o equivalente composto por uma fonte senoidal ligada em série com uma reatância. Esta última, calculada a partir da potência de curto-circuito no local do equivalente. Tanto a representação com base em fonte senoidal, quanto o cálculo da reatância são dados obtidos assumindo-se condição de regime permanente da rede elétrica.

No caso de estudo de transitórios, a fonte equivalente terá uma componente de regime transitória, além da principal que é de regime permanente. Haverá componente transitória, na situação em que a rede elétrica a ser reduzida (convertida em um equivalente), tiver elementos armazenadores de energia, como indutores e capacitores. Para ilustrar o problema, considere o circuito elétrico mostrado na Figura 1. Assume-se que entre o nó a e a referência (terra) poderá haver uma conexão, típica de uma falha de isolamento da rede, como um curto-circuito, ou mesmo uma carga. Uma maneira prática para calcular neste local a corrente de falta é obter o circuito equivalente de Thèvenin (ou de Norton) no ponto a. A simulação de um curto-circuito seria implementada, por exemplo, realizando-se a conexão de uma impedância nula entre o ponto a e o terra.

Para efeito de demonstração, considera-se que o circuito seja alimentado por uma fonte representada no domínio da frequência por $V_{0}(s)$. Para esse equivalente, calcula-se, inicialmente, a tensão equivalente de Thèvenin $V_{o c}(s)$ no ponto a do circuito.

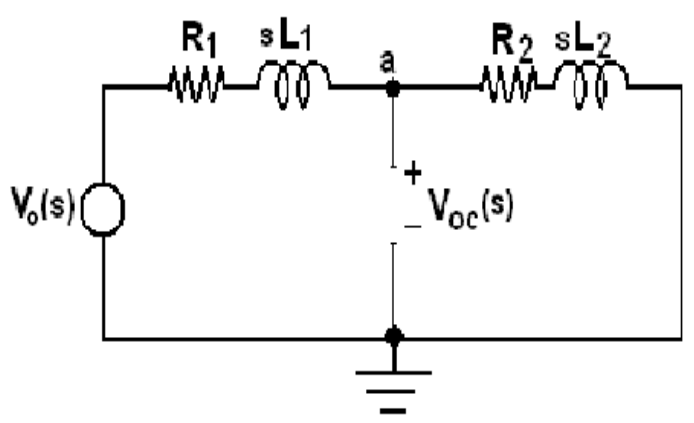

Figura 1: Circuito em que se calcula a tensão de circuito-aberto no nó a

Considerando-se que a tensão de alimentação do circuito no domínio do tempo é cossenoidal, do tipo

$$
v_{o}(t)=V_{m} \cos \left(\omega_{0} t\right),
$$

a transformada de Laplace de $v_{o}(t)$ é dada por

$$
V_{o}(s)=V_{m}
$$




$$
\begin{gathered}
K_{1} V_{m}=R B_{1}+A_{1} L \\
0=K_{1 a}+L B_{1} \\
0=K_{1 a} w_{0}^{2}+A_{1} R
\end{gathered}
$$

Resolvendo esse sistema linear obtém-se para $A_{1}$

$$
A_{1}=\underline{B_{1} L w^{2}}
$$


Proceeding Series of the Brazilian Society of Applied and Computational Mathematics, Vol. 1, N. 1, 2013.

$Y(s)=$

$\underline{V_{0}(s)}$ 
Os dados utilizados nas simulações são: $v_{0}(t)=10 \operatorname{sen}(5 t), R_{1}=1 \Omega, L_{1}=2 \mathrm{H}, C=1 \mathrm{~F}$, $R_{f}=0,05 \Omega, L_{f}=0,5 \mathrm{H}$.

Pode-se afirmar que para instantes inferiores a $t_{0}, V_{o c}$ funciona como se fosse a tensão de circuitoaberto do circuito equivalente de Thèvenin. No entanto, para instantes posteriores, o valor de $V_{o c}$ agora passa a representar a tensão instantânea sobre a carga $R_{f}+s L_{f}$.

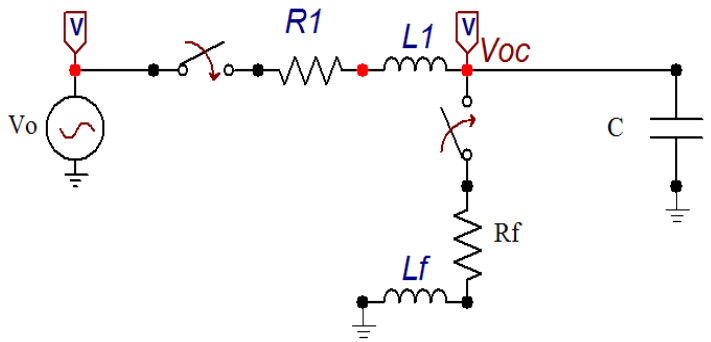

Figura 4: Carga conectada ao sistema no instante $t_{0}$ (domínio do tempo).

A simulação em questão foi feita no domínio do tempo para uma janela de dados de $20 \mathrm{~s}$, considerando-se passo de integração de 0,01 s. As simulações foram efetuadas nos aplicativos ATP (Alternative Transient Program) (Group, 1992) e Matlab. A tensão calculada a partir do circuito original é a indicada na Figura 5. Já a corrente fluindo pela carga é a indicada no gráfico da Figura 6.

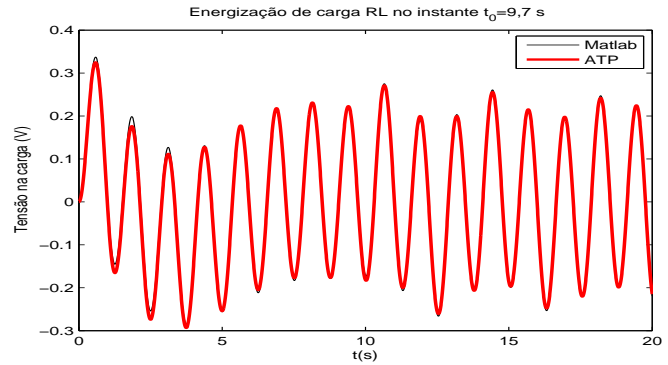

Figura 5: Tensão da carga calculada a partir do circuito original.

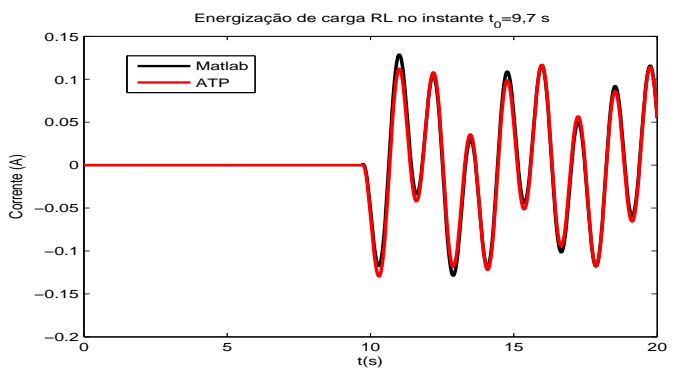

Figura 6: Corrente durante energização da carga calculada a partir do circuito original.

As Figuras 7 e 8 indicam a tensão $V_{o c}$ e a cor- rente na carga, calculadas a partir do equivalente dinâmico.

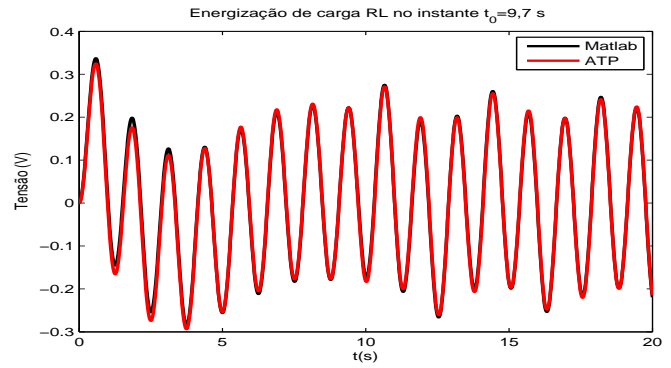

Figura 7: Tensão da carga calculada a partir do equivalente dinâmico de Thèvenin.

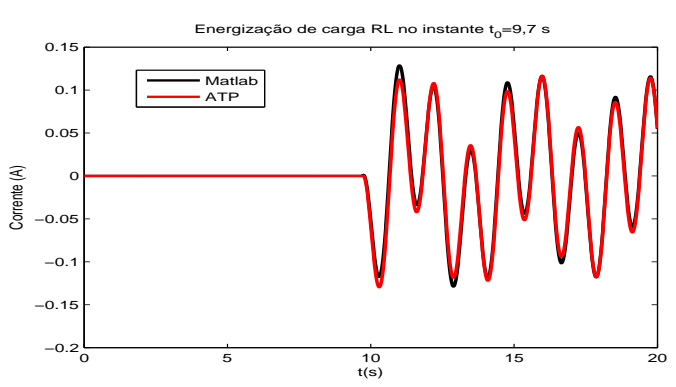

Figura 8: Corrente durante energização da carga calculada a partir do equivalente dinâmico de Thèvenin.

Foram efetuadas também simulações no domínio da frequência. Novamente, as variáveis de interesse são a corrente que flui pela carga e a tensão sobre este componente. No domínio da frequência, foram determinadas a corrente do equivalente de Norton e a tensão do equivalente de Thèvenin. Estes cálculos são obtidos diretamente do aplicativo ATP. Com estes dados, calcula-se a impedância (ou admitância) equivalente do circuito de Thèvenin (Norton).

Cálculos semelhantes foram efetuados utilizando o aplicativo Matlab. As Figuras 9 e 10 mostram a tensão de circuito-aberto e a corrente de curto-circuito franco no ponto onde a rede é apresentada na forma de um equivalente, respectivamente.

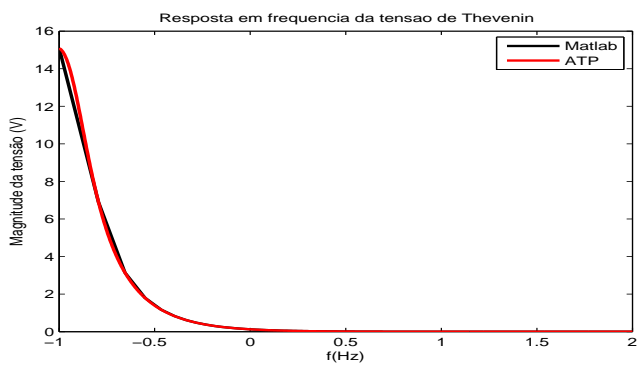

Figura 9: Tensão de Thèvenin. 


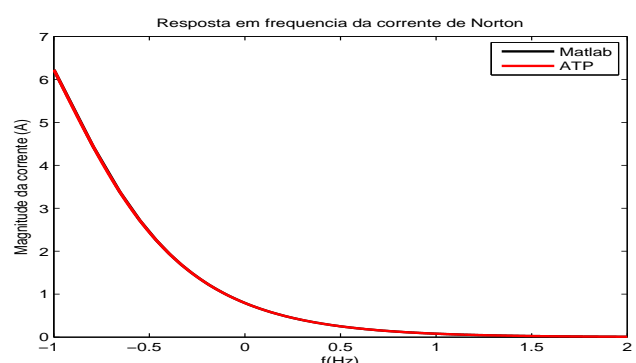

Figura 10: Corrente de Norton ou de curtocircuito franco no local de conexão da carga.

As Figuras 11 e 12, respectivamente, ilustram a magnitude e a fase da impedância calculadas.

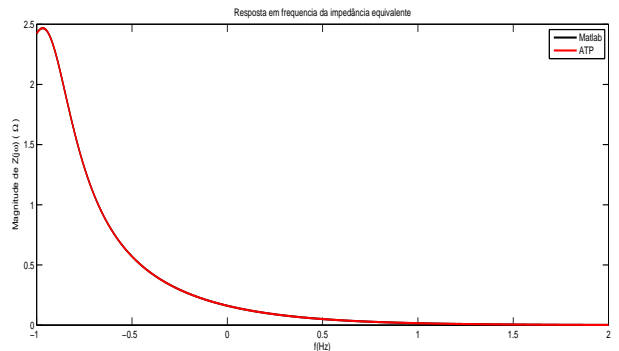

Figura 11: Magnitude da impedância equivalente.

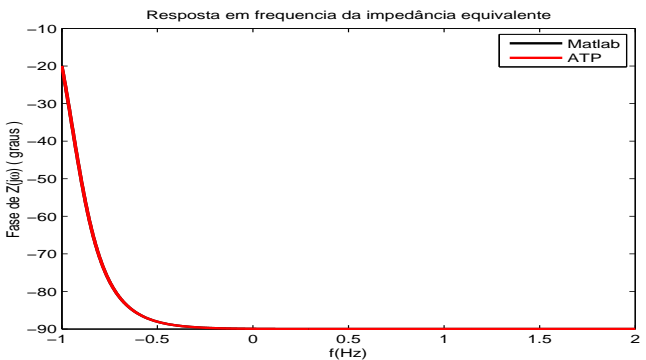

Figura 12: Fase da impedância equivalente.

Nos cálculos das respostas em frequência referentes à tensão e à corrente, foram utilizadas escalas de frequência diferentes propositalmente para se avaliar a precisão dos resultados. Notese que há diferença entre os valores do ATP (utilizado com mais pontos) (Group, 1992) e daqueles calculados via Matlab. No caso da resposta em frequência para se determinar a impedância, a mesma quantidade de pontos foi usada. Nesse caso, a diferença entre as curvas é imperceptível.

Note-se, mesmo com o chaveamento, tanto o resultado do cálculo da tensão quanto da corrente na carga tendem a ser muito próximos. Pode-se concluir então que com as informações no domínio da frequência é possível convertê-las para o domínio do tempo, tendo-se uma importante ferramenta computacional para realização de simulações que somente são acessíveis por meio do domínio do tempo. Esse é o caso quando se quer estudar a conexão de elementos não-lineares que ficam interconectados (Dounavis, 2000) ao circuito linear.

\section{Conclusões}

Neste artigo foi apresentada uma metodologia para cálculo do equivalente dinâmico de um circuito elétrico. A metodologia explora os resultados que são obtidos ao se utilizar os teoremas de Thévenin e de Norton. Foi proposto o cálculo de uma fonte equivalente que contém uma componente transitória e uma de regime permanente. A representação desta fonte é possível tanto no domínio do tempo quanto da frequência. A combinação dessa fonte com a impedância (admitância) dinâmica permite uma representação mais fiel do equivalente para estudos em que o objetivo é a análise do período transitório.

A metodologia pode ser estendida para sistemas de grande porte. No entanto, neste trabalho o interesse foi pela apresentação e dedução com detalhes das expressões com o intuito da formulação do problema.

\section{Agradecimentos}

O segundo autor agradece o apoio do DPP/UnB a esta pesquisa.

\section{Referências}

Carramaschi, I. H. C. (2010). Análise de equivalência em transitórios eletromagnéticos obtidos no domínio do tempo e da frequência, Master's thesis, Universidade de Brasília, Brasília.

Dounavis, A. (2000). Time domain macromodels for high speed interconnects, Master's thesis, Carlenton University, Ottawa, Ontario, Canada.

Freitas, F. D., Martins, N., Varricchio, S. L., Rommes, J. and Véliz, F. C. (2011). Reducedorder transfer matrices from rlc network descriptor models of electric power grids, IEEE Trans. on Power Systems 26: 1905-1916. DOI: 10.1109/TPWRS.2011.2136442

Group, E. E. U. (1992). Atp rule book., Technical report.

Johnson D. E., Hilburn, J. L. J. J. R. (1994). Fundamentos de análise de circuitos elétricos, Prentice-Hall do Brasil.

Watson, N. and Arrillaga, J. (2003). Power systems electromagnetic transients simulation, IEE, London, UK. DOI: 10.1049/PBPO039E 